\title{
CORRECTION
}

\section{Correction to: Augmentation in Healthcare Utilization of Patients with Opioid Use Disorder After Methadone Maintenance Treatment: A Retrospective Nationwide Study}

Chieh-Liang Huang · I-Ju Tsai • Wen-Chi Lin • Ing-Kang Ho •

Ruey-Yun Wang $\cdot$ Cynthia Wei-Sheng Lee (1)

Published online: May 5, 2021

(c) Springer Healthcare Ltd., part of Springer Nature 2021

Correction to: Adv Ther

https://doi.org/10.1007/s12325-021-01633-w

In the original article, the affiliation of the first author was published incorrectly. The correct affiliation is Ph.D. Program for Aging, College of

Medicine, China Medical University, Taichung, Taiwan.

The original article has been corrected.

The original article can be found online at https://doi. org/10.1007/s12325-021-01633-w.

C.-L. Huang · I.-K. Ho

Ph.D. Program for Aging, College of Medicine,

China Medical University, Taichung, Taiwan

C.-L. Huang

School of Medicine, China Medical University,

Taichung, Taiwan

C.-L. Huang

Tsaotun Psychiatric Center, Ministry of Health and Welfare, Nan-Tou County, Taiwan

I.-J. Tsai · W.-C. Lin · I.-K. Ho · C. W.-S. Lee ( $\square)$

Center for Drug Abuse and Addiction, China

Medical University Hospital, China Medical

University, Taichung, Taiwan

e-mail: T22529@mail.cmuh.org.tw;

cindywslee@gmail.com

I.-J. Tsai

Management Office for Health Data, China Medical University Hospital, China Medical University,

Taichung, Taiwan

I.-K. Ho · C. W.-S. Lee

Graduate Institute of Biomedical Sciences, China Medical University, Taichung, Taiwan

R.-Y. Wang

Department of Public Health, China Medical University, Taichung, Taiwan 